\title{
A real time immunoassay in alumina membranes
}

\author{
J. Álvarez \\ Materials \\ Science \\ Institute, \\ University of \\ Valencia, \\ Paterna, Spain \\ Email: \\ jesus2600@ho \\ tmail.com
}

\author{
M. Cretich M. J. Swann \\ ICRM, CNR, Farfield \\ Milano, Italy Group Ltd, \\ Email: Biolin \\ marina.cretich Scientific, \\ @icrm.cnr.it Manchester, \\ UK Email. \\ marcusswann \\ (a)outlook.com \\ K. B. \\ Gylfasson \\ KTH Royal \\ Institute of \\ Technology, \\ Stockholm, \\ Sweden \\ Email.
T. Volden
CSEM SA
Central
Switzerland,
Alpnach, \\ Switzerland \\ kristinn.gylfas \\ on@ee.kth.se
}

M. Chiari

D. Hill

ICRM, CNR,

Milano, Italy

marcella.chiar

i@icrm.cnr.it
Materials

Science

Institute,

University of

Valencia,

Paterna, Spain

Email:

daniel.hill@uv

.es

\begin{abstract}
To date, photonic biosensing with porous membranes has produced slow responses and long sensing times, due to the narrow (less than $100 \mathrm{~nm}$ ) closed end pores of the membranes used. Recently, polarimetry was used to demonstrate analyte flow through, and real time biosensing in, free-standing porous alumina membranes. Here, we demonstrate how an improved functionalization technology, has for the first time enabled a real-time immunoassay within a porous membrane with a total assay time below one hour. With the new approach, we show a noise floor for individual biosensing measurements of $3.7 \mathrm{ng} / \mathrm{ml}(25 \mathrm{pM})$, and a bulk refractive index detection limit of $5 \times 10^{-6}$ RIU, with a standard deviation of less than $5 \%$. The membranes, with their $200 \mathrm{~nm}$ pore diameter enabling targeted delivering of analytes to bioreceptors immobilized on the pore walls, therefore provide a route towards rapid and low cost realtime opto-fluidic biosensors for small sample volumes.
\end{abstract}

Keywords-Porous alumina; Form birefringence; Polarimetry; Optical biosensing; copolymer; quantum dots.

\section{INTRODUCTION}

With a higher surface area than planar biosensors for capturing analytes, and thus permitting lower detection limits [1], nanostructured materials like porous silicon (PSi) or

(a)

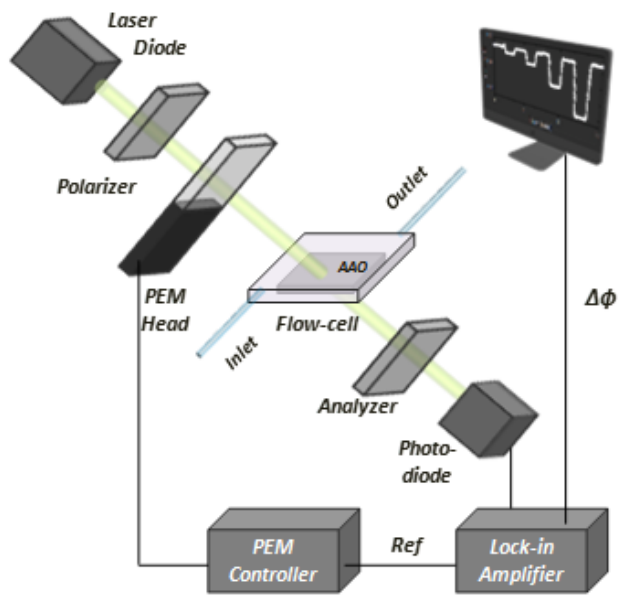

porous alumina (AAO) have gained special attention. PSi [2] [3] and AAO [4] [4] membrane optical biosensors however, produce slow responses and long sensing times, due to their narrow $(<100 \mathrm{~nm})$ closed end pores. An optical flow-through assay using a fibrous filter, has exposed the capture surface to the entire analyte solution to avoid mass transport limitations [5], but it was an ex-situ end point assay with complicated steps. Recently, free standing $200 \mathrm{~nm}$ pore AAO membranes, when functionalized with an epoxysilane, allowed analytes to flow-through less than $100 \mathrm{~nm}$ from the assay surface, breaking mass transport limitations and effectively targeting their delivery[6]. In that work, polarimetry (Fig 1) was used to take the preliminary real-time biosensing results.

Here, we report coating the membranes instead with a functional copolymer using a novel procedure, that has demonstrated less non-specific binding, and therefore greater selectivity, and more stability over time for immobilized allergens than epoxysilane [7]. Then, after measuring the bulk refractive index limit of detection we show the concentration performance of the immunoassay by using solutions of the first antibody at different concentrations, followed by a fixed concentration of the secondary antibody and streptavidin coated quantum dots.

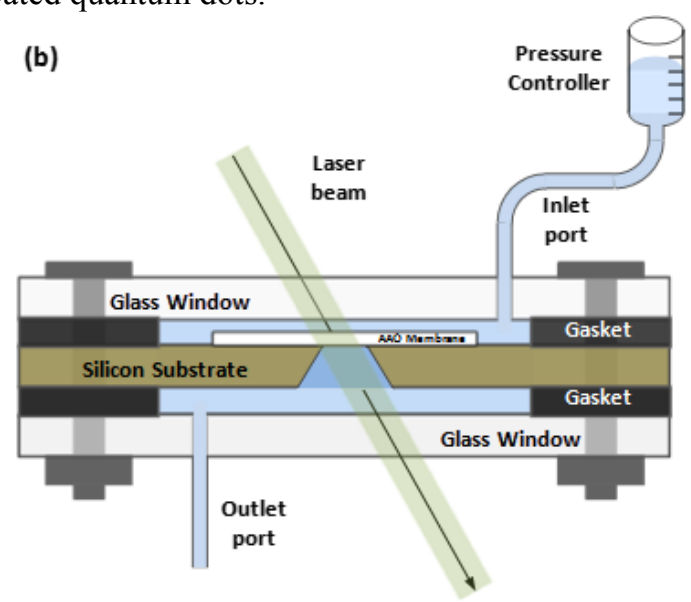

Figure 1(a): The optical polarimetric readout platform used for measuring the phase retardation within the membranes. (b) The fluidic setup is integrated within a flow-cell in which the mounted membrane is placed, and whose inlet port is connected to a pressure controller providing a constant pressure flow. 


\section{MATERIALS AND METHODS}

\section{A. Material}

The free-standing macroporous alumina membranes used within the experiments were purchased were manufactured by Whatman (Anodisc ${ }^{\mathrm{TM}}$ membranes, $13 \mathrm{~mm}$ diameter, $200 \mathrm{~nm}$ nominal pore diameter, $60 \mu \mathrm{m}$ thickness and 0.5 porosity). Prior to experiments, several were characterised morphologically by SEM to contrast the approximate pore dimensions against the nominal value. PBS (Phosphate buffered saline) tablets, Tween 20, toluene, dimethylformamide (DMF) N,N-dimethylacrylamide (DMA), [3-(methacryloyl-oxy)propyl]trimethoxysilane (MAPS), tetrahydrofuran (THF), azoisobutyonitrile (AIBN) and absolute ethanol, were acquired from Sigma-Aldrich (St. Louis, MO, USA). $N$-acryloyloxysuccinimide (NAS) was synthesized as reported elsewhere [9]. The bioreagents and their suppliers are listed elsewhere [7] purchased from Aldrich (St. Louis, MO, USA). All buffers were filtered through 0.2 um filters before use.

\section{B. Bioreceptor immobilization}

Due to its monomer composition poly(DMA-NAS-MAPS), first introduced in 2004 by Pirri and co-workers [10] provides a low fluorescence background for surfaces and a highly selective binding chemistry whilst retaining the native configuration of immobilized probes. This polymer has previously demonstrated the immobilization of allergens on different materials such as glass, nitrocellulose and silicon slides [11] and more recently on a SiOxNy Dual Polarization Interferometry chip [8]. This allowed the efficient measurement of their interactions with allergen-specific Immunoglobulin E (IgEs) in the complex matrix of serum, proving its suitability as a non-fouling coating and that it is robust to allergen storage. In this work following an oxygen plasma clean for 10 minutes in a Harrick Plasma cleaner (Ithaca, NY, USA) a functional coating of DMA and NAS was obtained via a novel two step procedure involving modification of the surface with MAPS followed by the grafting of poly(DMA-NAS) formed by in situ radical polymerization. Finally the membranes were washed in a Buchner funnel, under vacuum, first with DMF and then with THF, before being dried under vacuum at room temperature.

The allergen $\beta$-lactoglobulin $(1 \mathrm{mg} / \mathrm{mL}$ in PBS) was then spotted in a central area of the membranes to form a square of $5 \times 5 \mathrm{~mm}$ before being layed on nitrocellulose slides wetted with $10 \mu \mathrm{L}$ of the same antigen solution, so to assure complete filling of the pores by capillary action. They were then left overnight before being rinsed with $\mathrm{PBS}$ and remaining actives sites blocked with BSA $(0.1 \mathrm{mg} / \mathrm{mL})$. Full details can be found elsewhere [7].

\section{Membrane transfer to supports}

Following the immobilization of the bioreceptors onto the functionalized free-standing membranes for flow-through sensing experiments, the circular membranes were then transferred onto mechanical support chips. Details of the support chips chosen, due to their stability in an aqueous environment as well as the flat interface they provide for a good mechanical contact between them and the membranes, has previously been reported [7]. The edges of the $750 \mu \mathrm{m}$ diameter opening in the centre of each chip were chamfered on the reverse side in order to allow the laser light to readily pass through the hole without being occluded by the walls of the support chip. A $1 \mu \mathrm{m}$ thick layer of PMMA (Poly(methyl methacrylate)) resist was used as the adhesion layer and the supported membranes were then stored in a desiccator at $4{ }^{\circ} \mathrm{C}$.

\section{Readout platform}

The readout platform (Fig.1a) used a polarimetric setup [12] to interrogate the changes in the optical anisotropy of the macroporous free-standing membranes, the sensing mechanism for the bioassay. Analyte molecules within the pores modify the birefringence which is detected within the setup as a change of phase retardation between the components of light polarized along the main axis directions.

The fluidic setup uses a pressure controller connected to a syringe where the running buffer is stored (Fig.1b) to allow the analytes to be pumped through the flow cell encased and mechanically supported membranes. Before filling with liquid, the system is purged with $\mathrm{CO}_{2}$ for 5 minutes to ensure complete filling and to reduce the incidence of bubbles.

\section{RESULTS AND DISCUSSION}

\section{A. Volumetric sensing experiments}

In order to determine reproducibility for subsequent biosensing experiments a series of bulk refractive index experiments with the polarimetry setup were carried out, on ten different copolymer functionalised macroporous alumina membranes with an allergen immobilized on the pore surfaces. After flowing PBS-T through the membranes for fifteen minutes in order to obtain a base line, we flowed four solutions of $\mathrm{NaCl}$ in PBS with concentrations ranging from $0.2 \%(\mathrm{~m} / \mathrm{v})$ to $2 \%$. Fig. 2 (a) shows the measured polarimetric response for one of the membranes, whereas Fig. 2 (b) shows the overlaid phase retardation change for the ten different membranes, from the use of the different $\mathrm{NaCl}$ solutions. Fitting those values with a linear curve we obtain a mean sensitivity of 5.2 radians $\mathrm{RIU}^{-1}\left(\mathrm{rad} \mathrm{RIU}{ }^{-1}\right)$ with a standard deviation equal to $0.2 \mathrm{rad}$ RIU-1 (or 4\%), corresponding to a limit of detection of $5 \times 10^{-6}$ RIU from a measurement system resolution of $2.7 \times 10^{-5} \mathrm{rad}$ [12]. The mean sensitivity compares well to a theoretical bulk sensitivity of $5.311 \mathrm{rad} \mathrm{RIU}^{-1}$, calculated using the Bruggeman model [13], for a porous alumina membrane $60 \mu \mathrm{m}$ thick and with a 0.5 porosity if a refractive index of 1.65 is assumed for the alumina and 1.333 for the water in the cell. This suggests the pore size is still negligible compared to the wavelength, which would otherwise lead to a decrease in the observed sensitivity due to depolarization. 

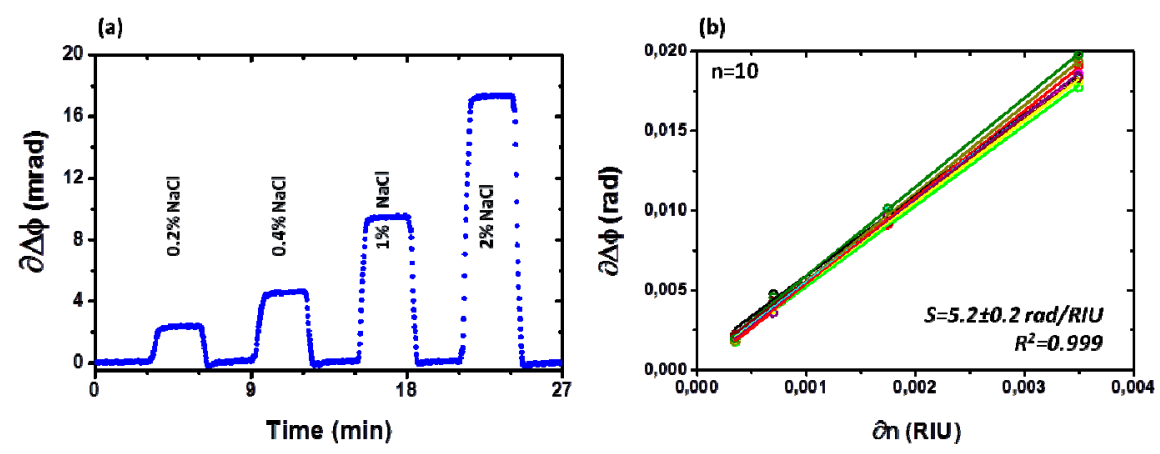

Figure 2 (a): A sensorgram of injections of solutions of varying concentration of $\mathrm{NaCl}$ in deionized water through a porous AAO membrane. (b) Overlaid phase retardation changes for ten different alumina membranes, as a function of refractive index changes from different $\mathrm{NaCl}$ concentrations.

\section{B. Immunosensing experiments}

After purching the flow-cell with $\mathrm{CO}_{2}$, a running buffer of PBS-T (PBS, $0.02 \%(\mathrm{v} / \mathrm{v})$ Tween 20) was introduced for 15 minutes before the behaviour of the immobilized allergen was tested by injecting its cognate antibody rabbit anti- $\beta$ lactoglobulin. The membrane was then rinsed again with the running buffer and the secondary antibody anti-rabbit-IgG (biotinylated) then introduced into the system followed by streptavidin conjugated quantum dots (SA-QD) as signal enhancers (Fig. 3), which increased the sensitivity for low concentrations of the primary antibody.

Fig. 4 shows the measured phase retardation when concentrations of $1 \mu \mathrm{g} / \mathrm{mL}(6.7 \mathrm{nM})$ are used for the first and secondary antibody and a concentration of $2.5 \mathrm{nM}$ is used for the SA-QD. Prior to the antibody injections, the baseline obtained during the buffer rinse showed good stability that demonstrated that the antigen is stably immobilized on the polymer coated surface. After recording a stable baseline during during six minutes we then injected the first antibody

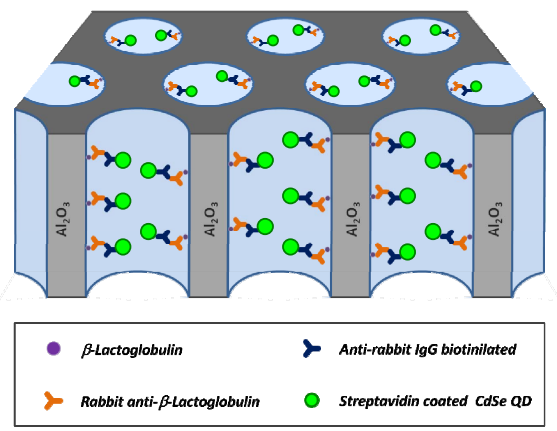

Figure 3: The immunoassay carried out in a macroporous alumina membrane. $\beta$-lactoglobulin protein was used as the immobilized antigen for the detection of rabbit anti- $\beta$-lactoglobulin. Biotinylated secondary antibody (anti-rabbitIgG) and streptavidin coated CdSe quantum dots were used to increase the signal produced by the primary antibody.

rabbit anti- $\beta$-lactoglobulin for a period of 10 minutes. Following a six minutes rinse with the running buffer the secondary antibody anti-rabbit IgG was then injected for 10 minutes. As the secondary antibody is polyclonal, a larger response is observed for this, compared to the binding of the initial primary antibody. After further rinsing, the SA-QDs were added as a signal enhancer at a concentration of $2.5 \mathrm{nM}$, which was sufficient to saturate the captured secondary antibodies. Due to the size of the SA-QDs an enhancement of 5 times is observed in the signal over the response produced by the secondary antibody. After this experiment a series of experiments was run using 12 different membranes with the same immunoassay where the concentration of the first antibody was varied between 5 and $1000 \mathrm{ng} / \mathrm{mL}$ in order to estimate the detection limit of the system - Fig. 4 (a) and Fig. 4 (b). The limit of detection of the system was taken as the analyte concentration that can reliably be distinguished from the noise [14]:

$$
L o D=L o B+1.645 \cdot S D_{\text {low_concentration_sample }}
$$

where $\mathrm{SD}_{\text {low_concentration_sample }}$ corresponds to the standard deviation obtained from a series of samples at low concentration and $L o B$ is the limit of blank defined as the highest analyte concentration value expected to be obtained for a sample containing no analytes:

$$
L o B=\text { mean }_{\text {blank }}+1.645 \cdot S D_{\text {blank }}
$$

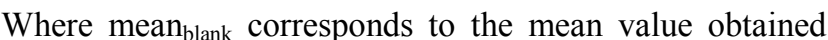
from a series of blank samples and SD corresponds to the standard deviation of the samples.

The $\mathrm{LoD}=33.7 \mathrm{ng} / \mathrm{ml}$, which is chiefly limited by the reproducibility of the mechanical setup rather than the measurement signal noise level, which would establish a limit an order of magnitude lower, at $3.7 \mathrm{ng} / \mathrm{mL}$.

Compared to porous membranes in flow-over configurations, the flow-through configuration enables the entire sample to pass through the membrane in close proximity to the immobilized recognition molecules enabling a more efficient convective delivery of analytes and so:

- $\quad$ an assay time of less than one hour for a sandwich assay employing both a secondary and tertiary binding, instead of 5 hours for a two-step assay [3].

- sample utilization is greatly improved, $1 \mathrm{~mL}$ of total analyte containing sample volume for a 10 minute injection versus a total volume of $50 \mathrm{~mL}(100$ minute injection at a flow rate of $0.5 \mathrm{~mL} / \mathrm{min}$ ) [3]. Or, $1 \mu \mathrm{g}$ ( $1 \mathrm{~mL}$ of $1 \mu \mathrm{g} / \mathrm{mL}$ Anti- $\beta$-lactoglobulin solution), compared to $5 \mathrm{mg}$ ( $50 \mathrm{~mL}$ of a $0.1 \mathrm{mg} / \mathrm{mL} \mathrm{IgG} \mathrm{solution).}$ 

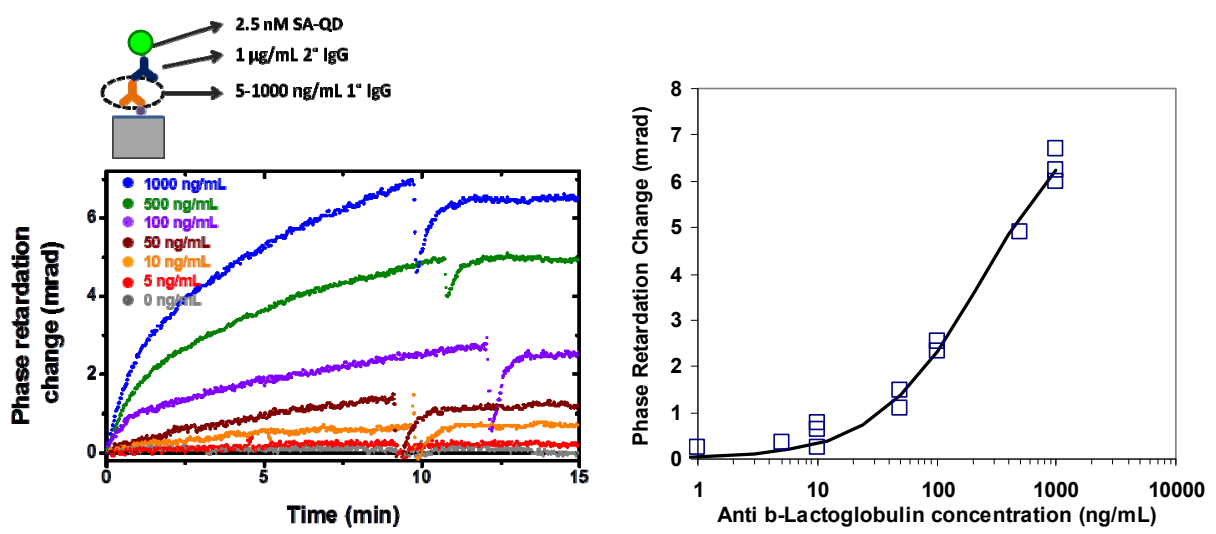

Figure 4 (a): An overlay of the signal responses produced by $2.5 \mathrm{nM}$ SA-QD injections on top of the secondary antibody (see inset), when the concentration of the primary antibody is increased from 5 to $1000 \mathrm{ng} / \mathrm{mL}$. (b) The corresponding phase retardation change, as a function of primary antibody concentration. The fitted line corresponds to a 1:1 binding model of $\mathrm{K}_{\mathrm{D}} 228 \mathrm{ng} / \mathrm{mL}$ and $\mathrm{R}_{\max }$ of $7.7 \mathrm{mrad}$.

\section{CONCLUSIONS AND FUTURE WORK}

A novel procedure to coat porous alumina membranes with a functional copolymer is presented, with less non-specific binding, and therefore greater selectivity, and more stability over time for immobilized allergens than epoxysilane.

A series of bulk refractive index experiments with immobilized allergens on the pore walls, yielded a detection limit of $5 \times 10^{-6}$ RIU, with a standard deviation $<5 \%$. Both the stability and the functionality of the coating and spotting procedures were then evaluated, with the response produced by the binding between the allergen protein and its cognate antibodies acquired in real-time. With the aid of SA-QD signal enhancers, a five-fold signal amplification compared with the signal produced by the secondary antibody alone was achieved, resulting in a noise floor for individual measurements of $3.7 \mathrm{ng} / \mathrm{ml}(25 \mathrm{pM})$ for a total assay time of under one hour. Compared to porous membrane biosensors in flow-over configurations, the flow-through approach reduces the assay time fivefold, and analyte consumption by three orders of magnitude.

The membranes, with their $200 \mathrm{~nm}$ pore diameters enable targeted delivering of analytes to bioreceptors immobilized on the pore walls, providing a route towards rapid and low cost real-time opto-fluidic biosensors for small sample volumes.

\section{ACKNOWLEDGMENT}

The authors would like to acknowledge the contributions from Geoff Platt (Farfield), Isabelle Metton (Phylogene), Steven Sievers (Charité) and Thomas Stadelmann (CSEM) for their participating in prior biosensing experiments that led up to this work. We also thank Helmut Knapp, Siegfried Graf (both CSEM) for the design and fabrication of the flow cell and Wouter van der Wijngaart (KTH) for fluidic discussions. Finally we thank Intenanomat for the use of their wet labs for reagent preparation and storage.

\section{REFERENCES}

[1] T.D. Lazzara, I. Mey, C. Steinem, A. Janshoff, "Benefits and limitations of porous substrates as biosensors for protein adsorption" Anal. Chem., Vol. 83(14), pp. 5624-5630 (2011).

[2] M.M. Orosco, C. Pacholski, M.J. Sailor "Real-time monitoring of enzyme activity in a mesoporous silicon double layer", Nat. Nanotechnol., Vol. 4, pp. 255-258 (2009).

[3] C.K. Tsang, T.L. Kelly, M.J. Sailor, Y.Y. Li, "Highly Stable Porous Silicon-Carbon Composites as Label-Free Optical Biosensors" ACS Nano Vol. 6, pp. 10546-10554 (2012).

[4] S.D. Alvarez, C.P. Li, C.E. Chiang, I.K. Schuller, M.J. Sailor, "A LabelFree Porous Alumina Interferometric Immunosensor" ACS Nano Vol. 3, pp. 3301-3307 (2009).

[5] T. Kumeria, M.D. Kurkuri, K.R. Diener, L. Parkinson, D. Losic, "Labelfree reflectometric interference microchip biosensor based on nanoporous alumina for detection of circulating tumour cells" Biosens. Bioelectron., Vol. 35(1), pp. 167-173 (2012).

[6] R. M. L. van Lieshout, T. van Domburg, M. Saalmink, R. Verbeek, R. Wimberger-Friedl, M. P. van Dieijen-Visser and C. Punyadeera ThreeDimensional Flow-Through Protein Platform, Anal. Chem. 2009, 81, 5165-5171

[7] J. Álvarez, L. Sola, G. Platt, M. Cretich, M. Swann, M. Chiari, D. Hill, and J. Martínez-Pastor, "Real-time polarimetric biosensing using macroporous alumina membranes" Proc. SPIE 8765, Bio-MEMS and Medical Microdevices, 87650I (May 28, 2013)

[8] G. W. Platt, F. Damin, M. J. Swann, I. Metton, G. Skorski, M. Cretich, M. Chiari, Allergen immobilisation and signal amplification by quantum dots for use in a biosensor assay of IgE in serum, Biosensors and Bioelectronics 52 (2014) 82-88

[9] M. Mammen, G. Dahmann, G.M. Whitesides, "Effective inhibitors of hemagglutination by influenza virus synthesized from polymers having active ester groups - insight into mechanism of inhibition", J. Med. Chem., 38 (1995), pp. 4179-4190

[10] Pirri, G ; Damin, F; Chiari, M; Bontempi, E; Depero, LE, "Characterization of a polymeric adsorbed coating for DNA microarray glass slides" Anal. Chem Volume: 76 Issue: 5 Pages: 1352-1358 (2004)

[11] Cretich, M., Breda, D., Damin, F., Borghi, M., Sola, L., Unlu, S. M., \& Chiari, M. (2010). Allergen microarrays on high-sensitivity silicon slides. Analytical and bioanalytical chemistry, 398(4), 1723-1733.

[12] J. Álvarez, C. Serrano, D. Hill, and J. Martínez-Pastor, "Real-time polarimetric optical sensor using macroporous alumina membranes," Opt. Lett. Vol. 38(7), pp. 1058-1060 (2013).

[13] T.C. Choy, "Effective Medium Theory, Principles and Applications," Oxford University Press, (1999).

[14] D.A. Armbruster and Terry Pry "Limit of Blank, Limit of Detection and Limit of Quantitation" Clin Biochem Rev, 29 (2008) 